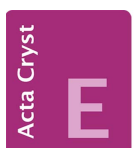

CRYSTALLOGRAPHIC COMMUNICATIONS

ISSN 2056-9890

Received 13 October 2015

Accepted 27 October 2015

Edited by M. Weil, Vienna University of Technology, Austria

Keywords: crystal structure; cobalt carbonyl; ammonia; hydrogen bonding

CCDC reference: 1433399

Supporting information: this article has supporting information at journals.iucr.org/e

\section{Crystal structure of $\left[\mathrm{Co}\left(\mathrm{NH}_{3}\right)_{6}\right]\left[\mathrm{Co}(\mathrm{CO})_{4}\right]_{2}$}

\author{
Thomas G. Müller and Florian Kraus*
}

Anorganische Chemie, Fluorchemie, Fachbereich Chemie, Philipps-Universität Marburg, Hans-Meerwein-Strasse 4, 35032 Marburg, Germany. *Correspondence e-mail: florian.kraus@chemie.uni-marburg.de

Hexaamminecobalt(II) bis[tetracarbonylcobaltate(-I)], $\left[\mathrm{Co}\left(\mathrm{NH}_{3}\right)_{6}\right]\left[\mathrm{Co}(\mathrm{CO})_{4}\right]_{2}$, was synthesized by reaction of liquid ammonia with $\mathrm{Co}_{2}(\mathrm{CO})_{8}$. The $\mathrm{Co}^{\mathrm{II}}$ atom is coordinated by six ammine ligands. The resulting polyhedron, the hexaamminecobalt(II) cation, exhibits point group symmetry $\overline{3}$. The $\mathrm{Co}^{-\mathrm{I}}$ atom is coordinated by four carbonyl ligands, leading to a tetracarbonylcobaltate $(-\mathrm{I})$ anion in the shape of a slightly distorted tetrahedron, with point group symmetry 3 . The crystal structure is related to that of high-pressure $\mathrm{BaC}_{2}$ (space group $R \overline{3} m)$, with the $\left[\mathrm{Co}\left(\mathrm{NH}_{3}\right)_{6}\right]^{2+}$ cations replacing the Ba sites and the $\left[\mathrm{Co}(\mathrm{CO})_{4}\right]^{-}$ anions replacing the $\mathrm{C}$ sites. $\mathrm{N}-\mathrm{H} \cdots \mathrm{O}$ hydrogen bonds between cations and anions stabilize the structural set-up in the title compound.

\section{Chemical context}

The reaction of $\mathrm{Co}_{2}(\mathrm{CO})_{8}$ with bases has already been described in the literature (Hieber et al., 1960). In addition, the reaction of dicobalt octacarbonyl with liquid ammonia has been known for several decades (Behrens \& Wakamatsu, 1966). Thereby $\mathrm{Co}_{2}(\mathrm{CO})_{8}$ forms with $\mathrm{NH}_{3}$ hexaamminecobalt(II) bis[tetracarbonylcobaltate(-I)], [Co( $\left.\left(\mathrm{NH}_{3}\right)_{6}\right]-$ $\left[\mathrm{Co}(\mathrm{CO})_{4}\right]_{2}$, which is obtained as orange air-sensitive crystals. During this reaction, $\mathrm{CO}$ is released and reacts with ammonia to urea. However, structural data of of the title compound were missing and are presented in this communication.

\section{Structural commentary}

The cobalt atom Co1 of the hexaamminecobalt(II) cation occupies Wyckoff position $3 a$ with site symmetry $\overline{3}$. It is coordinated by six symmetry-related ammine ligands in form of a slightly distorted octahedron. The $\mathrm{Co}-\mathrm{N}$ distance in the $\left[\mathrm{Co}\left(\mathrm{NH}_{3}\right)_{6}\right]$ octahedron is 2.1876 (16) $\AA$ 年 which compares well
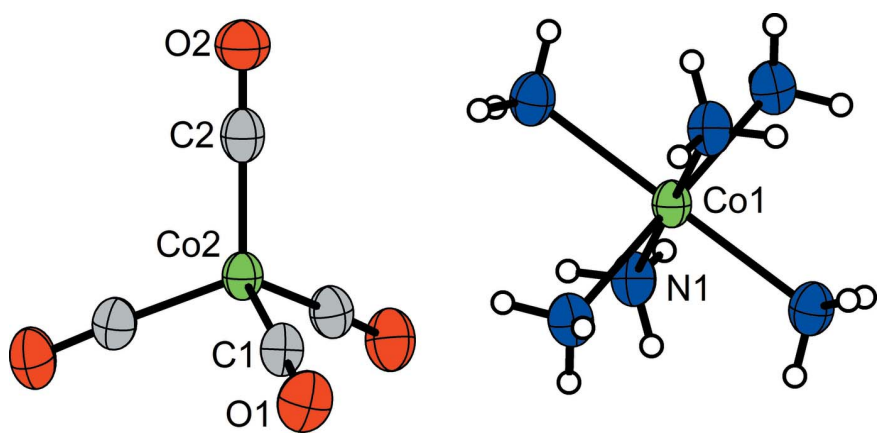

Figure 1

The molecular structures of the tetracarbonylcobaltate $(-\mathrm{I})$ anion and of the hexaamminecobalt(II) cation of the title compound. Displacement ellipsoids are shown at the $70 \%$ probability level. Labelling of symmetryequivalent atoms has been omitted for clarity. 


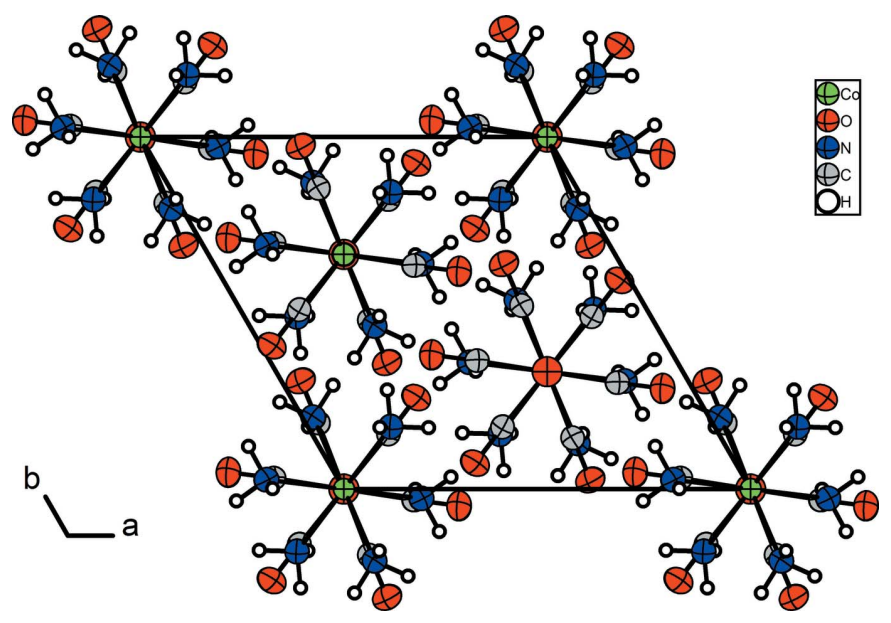

Figure 2

The unit cell of $\left[\mathrm{Co}\left(\mathrm{NH}_{3}\right)_{6}\right]\left[\mathrm{Co}(\mathrm{CO})_{4}\right]_{2}$, viewed along [001]. Displacement ellipsoids are shown at the $70 \%$ probability level.

with those of other reported hexaamminecobalt(II) structures (Barnet et al., 1966).

The cobalt atom $\mathrm{Co} 2$ of the tetracarbonylcobaltate(-I) anion occupies Wyckoff position $6 c$ and exhibits site symmetry 3 .. It is coordinated by four carbonyl ligands in a shape close to an ideal tetrahedron. The distances between the $\mathrm{Co} 2$ atom and the carbon atoms $\mathrm{C} 1$ and $\mathrm{C} 2$ of the ligands are 1.7664 (18) and 1.779 (3) $\AA$, respectively. In the literature, distances in the range from 1.77 (2) to 1.82 (2) $\AA$ are reported for $\mathrm{Co}-\mathrm{C}$ in the compound $\mathrm{Co}_{2}(\mathrm{CO})_{8}$ (Sumner et al., 1964). In the carbonyl ligands, the observed distances are in the expected range with 1.153 (2) and 1.140 (4) $\AA$ for $\mathrm{C} 1-\mathrm{O} 1$ and $\mathrm{C} 2-\mathrm{O} 2$, respectively. For the compound $\mathrm{Co}_{2}(\mathrm{CO})_{8}$ distances from 1.14 (2) to 1.33 (2) A were reported (Sumner et al., 1964).

The crystal structure of $\left[\mathrm{Co}\left(\mathrm{NH}_{3}\right)_{6}\right]\left[\mathrm{Co}(\mathrm{CO})_{4}\right]_{2}$ can be derived from the high-pressure rhombohedral phase of $\mathrm{BaC}_{2}$ $\left(\mathrm{BaC}_{2}-\mathrm{HP} 1, R \overline{3} m\right)$ (Efthimiopoulos et al., 2012). Formally, the $\mathrm{Ba}$ sites on Wyckoff position $3 a$ are replaced by the hexaammine cobalt(II) octahedra and the $\mathrm{C}$ site on position $6 c$ is replaced by the tetracarbonylcobaltate $(-\mathrm{I})$ tetrahedron.

The molecular components of the title compound are shown in Fig. 1. The unit cell of $\left[\mathrm{Co}\left(\mathrm{NH}_{3}\right)_{6}\right]\left[\mathrm{Co}(\mathrm{CO})_{4}\right]_{2}$ projected along [001] is shown in Fig. 2.

\section{Supramolecular features}

The arrangement of $\left[\mathrm{Co}\left(\mathrm{NH}_{3}\right)_{6}\right]^{2+}$ octahedra and $\left[\mathrm{Co}(\mathrm{CO})_{4}\right]^{-}$ tetrahedra in the crystal structure is stabilized by $\mathrm{N}-\mathrm{H} \cdots \mathrm{O}$ hydrogen bonds with the $\mathrm{N} 1$ atom as donor and the oxygen atoms $\mathrm{O} 1$ and $\mathrm{O} 2$ as acceptors atoms. One of the hydrogen bonds $(\mathrm{N}-\mathrm{H} 1 \mathrm{C})$ is forked while, remarkably, in the neigh-

$3 \mathrm{Co}_{2}(\mathrm{CO})_{8}+12 \mathrm{NH}_{3} \longrightarrow 2\left[\mathrm{Co}\left(\mathrm{NH}_{3}\right)_{6}\right]\left[\mathrm{Co}(\mathrm{CO})_{4}\right]_{2}+8 \mathrm{CO}$

Figure 3

Reaction equation for the preparation of the title compound.

Table 1

Hydrogen-bond geometry $\left(\AA,^{\circ}\right)$.

\begin{tabular}{lllll}
\hline$D-\mathrm{H} \cdots A$ & $D-\mathrm{H}$ & $\mathrm{H} \cdots A$ & $D \cdots A$ & $D-\mathrm{H} \cdots A$ \\
\hline $\mathrm{N} 1-\mathrm{H} 1 A \cdots \mathrm{O} 1^{\mathrm{i}}$ & $0.87(4)$ & $2.49(4)$ & $3.159(2)$ & $135(3)$ \\
$\mathrm{N} 1-\mathrm{H} 1 C \cdots \mathrm{O} 1^{\mathrm{ii}}$ & $0.87(3)$ & $2.59(3)$ & $3.290(2)$ & $138(3)$ \\
$\mathrm{N} 1-\mathrm{H} 1 C \cdots \mathrm{O} 2^{\mathrm{iii}}$ & $0.87(3)$ & $2.49(3)$ & $3.249(3)$ & $146(3)$ \\
\hline $\begin{array}{l}\text { Symmetry codes: } \\
x+\frac{2}{3}, y+\frac{1}{3}, z+\frac{1}{3} .\end{array}$ & & & &
\end{tabular}

bourhood of the hydrogen atom $\mathrm{H} 1 B$ no acceptor atom in the range of the sum of the van der Waals radii is present. Detailed information about hydrogen-bonding distances and angles are given in Table 1.

\section{Synthesis and crystallization}

$86 \mathrm{mg}(29.4 \mathrm{mmol})$ of $\mathrm{Co}_{2}(\mathrm{CO})_{8}$ were placed in a flame-dried bomb tube under argon. $0.2 \mathrm{ml}$ of liquid ammonia were condensed to the bomb tube. The bomb tube, now containing an orange solution, was flame-sealed and stored at room temperature. The reaction equation is given in Fig. 3. After six months of crystallization time, moisture- and temperaturesensitive, orange single crystals of the title compound were obtained in almost quantitative yield from the still orange solution. After manual separation of the crystals under a lightoptical microscope and evaporation of the solvent only a minute orange residue remained.

Table 2

Experimental details.

Crystal data

Chemical formula

$M_{\text {r }}$

Crystal system, space group

Temperature (K)

$a, c(\AA)$

$V\left(\AA^{3}\right)$

Z

Radiation type

$\mu\left(\mathrm{mm}^{-1}\right)$

Crystal size (mm)

Data collection

Diffractometer

Absorption correction

$T_{\min }, T_{\max }$

No. of measured, independent and observed $[I>2 \sigma(I)]$ reflections

$R_{\text {int }}$

$(\sin \theta / \lambda)_{\max }\left(\AA^{-1}\right)$

Refinement

$R\left[F^{2}>2 \sigma\left(F^{2}\right)\right], w R\left(F^{2}\right), S$

No. of reflections

No. of parameters

$\mathrm{H}$-atom treatment

$\Delta \rho_{\max }, \Delta \rho_{\min }\left(\mathrm{e} \AA^{-3}\right)$

Computer programs: $X$-AREA (Stoe \& Cie, 2011), X-RED32 (Stoe \& Cie, 2009), SHELXT (Sheldrick, 2015a), SHELXLE (Hübschle et al., 2011) and SHELXL2014 (Sheldrick, 2015b), DIAMOND (Brandenburg, 2015) and publCIF (Westrip, 2010). 


\section{Refinement}

Crystal data, data collection and structure refinement details are summarized in Table 2. All hydrogen atoms of the ammine ligands were located from a difference Fourier map and were refined isotropically without any further restraints.

\section{Acknowledgements}

FK thanks the Deutsche Forschungsgemeinschaft for his Heisenberg professorship.

\section{References}

Barnet, M. T., Craven, B. M., Freeman, H. C., Kime, N. E. \& Ibers, J. A. (1966). Chem. Commun. (London), 10, 307-308.

Behrens, H. \& Wakamatsu, H. (1966). Chem. Ber. 99, 2753-2756.
Brandenburg, K. (2015). DIAMOND. Crystal Impact GbR, Bonn, Germany.

Efthimiopoulos, I., Kunc, K., Vazhenin, G. V., Stavrou, E., Syassen, K., Hanfland, M., Liebig, S. \& Ruschewitz, U. (2012). Phys. Rev. B, $\mathbf{8 5}, 054105$.

Hieber, W., Beck, W. \& Braun, G. (1960). Angew. Chem. 72, 795-801. Hübschle, C. B., Sheldrick, G. M. \& Dittrich, B. (2011). J. Appl. Cryst. 44, 1281-1284.

Sheldrick, G. M. (2015a). Acta Cryst. A71, 3-8.

Sheldrick, G. M. (2015b). Acta Cryst. C71, 3-8.

Stoe \& Cie (2009). X-RED32 and X-SHAPE. Stoe \& Cie GmbH, Darmstadt, Germany.

Stoe \& Cie (2011). X-AREA. Stoe \& Cie GmbH, Darmstadt, Germany.

Sumner, G. G., Klug, H. P. \& Alexander, L. E. (1964). Acta Cryst. 17, 732-742.

Westrip, S. P. (2010). J. Appl. Cryst. 43, 920-925. 


\section{supporting information}

Acta Cryst. (2015). E71, 1418-1420 [https://doi.org/10.1107/S2056989015020290]

\section{Crystal structure of $\left[\mathrm{Co}\left(\mathrm{NH}_{3}\right)_{6}\right]\left[\mathrm{Co}(\mathrm{CO})_{4}\right]_{2}$}

\section{Thomas G. Müller and Florian Kraus}

\section{Computing details}

Data collection: $X$-AREA (Stoe \& Cie, 2011); cell refinement: $X$-AREA (Stoe \& Cie, 2011); data reduction: $X$-RED32 (Stoe \& Cie, 2009); program(s) used to solve structure: SHELXT (Sheldrick, 2015a); program(s) used to refine structure: SHELXLE (Hübschle et al., 2011) and SHELXL2014 (Sheldrick, 2015b); molecular graphics: DIAMOND (Brandenburg, 2015); software used to prepare material for publication: publCIF (Westrip, 2010).

Hexaamminecobalt(II) bis[tetracarbonylcobaltate(-I)]

Crystal data

$\left[\mathrm{Co}\left(\mathrm{NH}_{3}\right)_{6}\right]\left[\mathrm{Co}(\mathrm{CO})_{4}\right]_{2}$

$M_{r}=503.07$

Trigonal, $R \overline{3}$

$a=9.3679(4) \AA$

$c=18.3089(18) \AA$

$V=1391.48(18) \AA^{3}$

$Z=3$

$F(000)=759$

Data collection

Stoe IPDS-2T

diffractometer

Radiation source: sealed X-ray tube, $12 \times 0.4$ $\mathrm{mm}$ long-fine focus

Plane graphite monochromator

Detector resolution: 6.67 pixels $\mathrm{mm}^{-1}$

rotation method scans

Absorption correction: integration

(X-RED32 and X-SHAPE; Stoe \& Cie, 2009)

Refinement

Refinement on $F^{2}$

Least-squares matrix: full

$R\left[F^{2}>2 \sigma\left(F^{2}\right)\right]=0.034$

$w R\left(F^{2}\right)=0.090$

$S=1.08$

994 reflections

52 parameters

0 restraints

Hydrogen site location: difference Fourier map

All H-atom parameters refined
$D_{\mathrm{x}}=1.801 \mathrm{Mg} \mathrm{m}^{-3}$

Mo $K \alpha$ radiation, $\lambda=0.71073 \AA$

Cell parameters from 15618 reflections

$\theta=3.3-33.4^{\circ}$

$\mu=2.70 \mathrm{~mm}^{-1}$

$T=100 \mathrm{~K}$

Block, orange

$0.16 \times 0.12 \times 0.08 \mathrm{~mm}$

$T_{\min }=0.649, T_{\max }=0.907$

7025 measured reflections

994 independent reflections

910 reflections with $I>2 \sigma(I)$

$R_{\text {int }}=0.087$

$\theta_{\text {max }}=31.0^{\circ}, \theta_{\text {min }}=3.3^{\circ}$

$h=-13 \rightarrow 13$

$k=-13 \rightarrow 13$

$l=-26 \rightarrow 26$

$w=1 /\left[\sigma^{2}\left(F_{\mathrm{o}}^{2}\right)+(0.0529 P)^{2}+1.0515 P\right]$

where $P=\left(F_{\mathrm{o}}^{2}+2 F_{\mathrm{c}}^{2}\right) / 3$

$(\Delta / \sigma)_{\max }<0.001$

$\Delta \rho_{\max }=0.87 \mathrm{e} \AA^{-3}$

$\Delta \rho_{\min }=-0.65$ e $\AA^{-3}$

Extinction correction: SHELXL2014

(Sheldrick, 2015),

$\mathrm{Fc}^{*}=\mathrm{kFc}\left[1+0.001 \mathrm{xFc}^{2} \lambda^{3} / \sin (2 \theta)\right]^{-1 / 4}$

Extinction coefficient: 0.0040 (8) 


\section{Special details}

Geometry. All e.s.d.'s (except the e.s.d. in the dihedral angle between two 1.s. planes) are estimated using the full covariance matrix. The cell e.s.d.'s are taken into account individually in the estimation of e.s.d.'s in distances, angles and torsion angles; correlations between e.s.d.'s in cell parameters are only used when they are defined by crystal symmetry. An approximate (isotropic) treatment of cell e.s.d.'s is used for estimating e.s.d.'s involving l.s. planes.

Fractional atomic coordinates and isotropic or equivalent isotropic displacement parameters $\left(\hat{A}^{2}\right)$

\begin{tabular}{lllll}
\hline & $x$ & $y$ & $z$ & $U_{\text {iso }} * / U_{\text {eq }}$ \\
\hline Co1 & 0.0000 & 0.0000 & 0.0000 & $0.01863(18)$ \\
Co2 & -0.6667 & -0.3333 & $0.04221(2)$ & $0.01972(17)$ \\
O1 & $-0.61903(19)$ & $-0.02591(18)$ & $0.10467(9)$ & $0.0315(3)$ \\
O2 & -0.6667 & -0.3333 & $-0.11725(14)$ & $0.0298(5)$ \\
N1 & $-0.0266(2)$ & $-0.2037(2)$ & $0.06820(9)$ & $0.0245(3)$ \\
C1 & $-0.6354(2)$ & $-0.1451(2)$ & $0.07846(10)$ & $0.0231(3)$ \\
C2 & -0.6667 & -0.3333 & $-0.05497(19)$ & $0.0237(5)$ \\
H1A & $-0.121(5)$ & $-0.295(5)$ & $0.0656(19)$ & $0.054(10)^{*}$ \\
H1B & $0.034(4)$ & $-0.247(4)$ & $0.0558(17)$ & $0.038(7)^{*}$ \\
H1C & $-0.001(4)$ & $-0.176(4)$ & $0.1135(19)$ & $0.043(8)^{*}$ \\
\hline
\end{tabular}

Atomic displacement parameters $\left(\AA^{2}\right)$

\begin{tabular}{lllllll}
\hline & $U^{11}$ & $U^{22}$ & $U^{33}$ & $U^{12}$ & $U^{13}$ & $U^{23}$ \\
\hline Co1 & $0.0160(2)$ & $0.0160(2)$ & $0.0240(3)$ & $0.00799(10)$ & 0.000 & 0.000 \\
Co2 & $0.01726(19)$ & $0.01726(19)$ & $0.0247(3)$ & $0.00863(9)$ & 0.000 & 0.000 \\
O1 & $0.0323(7)$ & $0.0236(7)$ & $0.0410(8)$ & $0.0158(6)$ & $-0.0025(6)$ & $-0.0036(5)$ \\
O2 & $0.0316(8)$ & $0.0316(8)$ & $0.0262(12)$ & $0.0158(4)$ & 0.000 & 0.000 \\
N1 & $0.0209(7)$ & $0.0212(7)$ & $0.0307(7)$ & $0.0101(6)$ & $-0.0002(5)$ & $0.0015(5)$ \\
C1 & $0.0192(7)$ & $0.0203(7)$ & $0.0292(8)$ & $0.0095(6)$ & $-0.0007(6)$ & $0.0009(6)$ \\
C2 & $0.0198(8)$ & $0.0198(8)$ & $0.0317(15)$ & $0.0099(4)$ & 0.000 & 0.000 \\
\hline
\end{tabular}

Geometric parameters $\left(\AA,{ }^{\circ}\right)$

\begin{tabular}{|c|c|c|c|}
\hline $\mathrm{Co} 1-\mathrm{N} 1^{\mathrm{i}}$ & $2.1876(16)$ & $\mathrm{Co} 2-\mathrm{C} 1$ & 1.7664 (18) \\
\hline $\mathrm{Co} 1-\mathrm{N} 1^{\mathrm{ii}}$ & $2.1876(16)$ & $\mathrm{Co} 2-\mathrm{C}^{\mathrm{vi}}$ & $1.7664(18)$ \\
\hline $\mathrm{Co} 1-\mathrm{N} 1^{\mathrm{iii}}$ & $2.1876(16)$ & $\mathrm{Co} 2-\mathrm{C} 1^{\mathrm{vii}}$ & $1.7664(18)$ \\
\hline $\mathrm{Co} 1-\mathrm{N} 1^{\text {iv }}$ & $2.1876(16)$ & $\mathrm{Co} 2-\mathrm{C} 2$ & $1.779(3)$ \\
\hline $\mathrm{Co} 1-\mathrm{N} 1$ & $2.1877(16)$ & $\mathrm{O} 1-\mathrm{C} 1$ & $1.153(2)$ \\
\hline $\mathrm{Co} 1-\mathrm{N} 1^{\mathrm{v}}$ & $2.1877(16)$ & $\mathrm{O} 2-\mathrm{C} 2$ & $1.140(4)$ \\
\hline $\mathrm{N} 1^{\mathrm{i}}-\mathrm{Co} 1-\mathrm{N} 1^{\mathrm{ii}}$ & $180.00(9)$ & 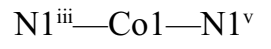 & $90.65(6)$ \\
\hline $\mathrm{N} 1^{\mathrm{i}}-\mathrm{Co} 1-\mathrm{N} 1^{\mathrm{iii}}$ & $90.65(6)$ & $\mathrm{N} 1^{\mathrm{iv}}-\mathrm{Co} 1-\mathrm{N} 1^{\mathrm{v}}$ & $89.35(6)$ \\
\hline $\mathrm{N} 1^{\mathrm{ii}}-\mathrm{Co} 1-\mathrm{N} 1^{\mathrm{iii}}$ & $89.35(6)$ & $\mathrm{N} 1-\mathrm{Co} 1-\mathrm{N} 1^{\mathrm{v}}$ & 180.0 \\
\hline $\mathrm{N} 1-\mathrm{Co} 1-\mathrm{N} 1^{\mathrm{iv}}$ & $89.35(6)$ & $\mathrm{C} 1-\mathrm{Co} 2-\mathrm{C}^{\mathrm{vi}}$ & $106.76(7)$ \\
\hline 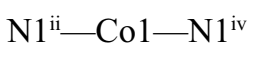 & $90.65(6)$ & $\mathrm{C} 1-\mathrm{Co} 2-\mathrm{C} 1^{\mathrm{vii}}$ & $106.75(7)$ \\
\hline $\mathrm{N} 1^{\mathrm{iii}}-\mathrm{Co} 1-\mathrm{N} 1^{\mathrm{iv}}$ & $180.00(11)$ & $\mathrm{C} 1^{\mathrm{vi}}-\mathrm{Co} 2-\mathrm{C} 1^{\mathrm{vii}}$ & $106.75(7)$ \\
\hline $\mathrm{N} 1{ }^{\mathrm{i}}-\mathrm{Co} 1-\mathrm{N} 1$ & $89.35(6)$ & $\mathrm{C} 1-\mathrm{Co} 2-\mathrm{C} 2$ & $112.07(6)$ \\
\hline $\mathrm{N} 1 \mathrm{ii}-\mathrm{Co} 1-\mathrm{N} 1$ & $90.65(6)$ & $\mathrm{C} 1^{\mathrm{vi}}-\mathrm{Co} 2-\mathrm{C} 2$ & $112.07(6)$ \\
\hline
\end{tabular}




\begin{tabular}{|c|c|c|c|}
\hline $\mathrm{N} 1^{\mathrm{iii}}-\mathrm{Co} 1-\mathrm{N} 1$ & $89.35(6)$ & $\mathrm{C} 1 \mathrm{vii}-\mathrm{Co} 2-\mathrm{C} 2$ & $112.07(6)$ \\
\hline $\mathrm{N} 1{ }^{\mathrm{iv}}-\mathrm{Co} 1-\mathrm{N} 1$ & $90.65(6)$ & $\mathrm{O} 1-\mathrm{C} 1-\mathrm{Co} 2$ & 177.07 (17) \\
\hline $\mathrm{N} 1^{\mathrm{i}}-\mathrm{Co} 1-\mathrm{N} 1^{\mathrm{v}}$ & $90.65(6)$ & $\mathrm{O} 2-\mathrm{C} 2-\mathrm{Co} 2$ & 180.0 \\
\hline $\mathrm{N} 1^{\mathrm{ii}}-\mathrm{Co} 1-\mathrm{N} 1^{\mathrm{v}}$ & $89.35(6)$ & & \\
\hline
\end{tabular}

Symmetry codes: (i) $x-y, x,-z$; (ii) $-x+y,-x$, $z$; (iii) $y,-x+y,-z$; (iv) $-y, x-y, z$; (v) $-x,-y,-z$; (vi) $-y-1, x-y, z$; (vii) $-x+y-1,-x-1, z$.

Hydrogen-bond geometry $\left(A,{ }^{\circ}\right)$

\begin{tabular}{lllll}
\hline$D-\mathrm{H} \cdots A$ & $D-\mathrm{H}$ & $\mathrm{H} \cdots A$ & $D \cdots A$ & $D-\mathrm{H}^{\cdots} A$ \\
\hline $\mathrm{N} 1-\mathrm{H} 1 A \cdots \mathrm{O} 1^{\text {vii }}$ & $0.87(4)$ & $2.49(4)$ & $3.159(2)$ & $135(3)$ \\
$\mathrm{N} 1-\mathrm{H} 1 C \cdots \mathrm{O} 1^{\text {viii }}$ & $0.87(3)$ & $2.59(3)$ & $3.290(2)$ & $138(3)$ \\
$\mathrm{N} 1-\mathrm{H} 1 C \cdots \mathrm{O} 2^{\text {ix }}$ & $0.87(3)$ & $2.49(3)$ & $3.249(3)$ & $146(3)$
\end{tabular}

Symmetry codes: (vii) $-x+y-1,-x-1, z$; (viii) $x-y+2 / 3, x+1 / 3,-z+1 / 3$; (ix) $x+2 / 3, y+1 / 3, z+1 / 3$. 